\title{
Evaluating differences in the clinical impact of a free online weight loss programme, a resource-intensive commercial weight loss programme and an active control condition: a parallel randomised controlled trial
}

Aidan Q. Innes ${ }^{1,2}$, Greig Thomson ${ }^{1}$, Mary Cotter ${ }^{1}$, James A. King ${ }^{3,4}$, Niels B. J. Vollaard ${ }^{5}$ and Benjamin M. Kelly ${ }^{1,2^{*}}$

\begin{abstract}
Background: Finding effective intervention strategies to combat rising obesity levels could significantly reduce the burden that obesity and associated non-communicable diseases places on both individuals and the National Health Service.

Methods: In this parallel randomised-controlled trial, 76 participants who are overweight or obese (50 female) were given free access to a fitness centre for the duration of the 12-week intervention and randomised to one of three interventions. The commercial intervention, the Healthy Weight Programme, (HWP, $n=25,10 / 15$ men/women) consisted of twelve 1-h nutrition coaching sessions with a nutritionist delivered as a mixture of group and 1 to 1 sessions. In addition, twice-weekly exercise sessions (24 in total) were delivered by personal trainers for 12 weeks. The NHS intervention ( $n=25,8 / 17$ men/women) consisted of following an entirely self-managed 12-week online NHS resource. The GYM intervention ( $n=26,8 / 18$ men/women) received no guidance or formal intervention. All participants were provided with a gym induction for safety and both the NHS and GYM participants were familiarised with ACSM physical activity guidelines by way of a hand-out.

Results: The overall follow-up rate was $83 \%$. Body mass was significantly reduced at post-intervention in all groups (HWP: $N=18,-5.17 \pm 4.22 \mathrm{~kg}$, NHS: $N=21-4.19 \pm 5.49 \mathrm{~kg}$; GYM: $N=24-1.17 \pm 3.00 \mathrm{~kg} ; p<0.001$ ) with greater reductions observed in HWP and NHS groups compared to GYM $(p<0.05)$. Out with body mass and BMI, there were no additional statistically significant time $x$ intervention interaction effects.

Conclusions: This is the first study to evaluate the efficacy of both a free online NHS self-help weight-loss tool and a commercial weight loss programme that provides face-to-face nutritional support and supervised exercise. The findings suggest that both interventions are superior to an active control condition with regard to eliciting shortterm weight-loss.
\end{abstract}

Trial registration: ISRCTN Registry - ISRCTN31489026. Prospectively registered: 27/07/16.

Keywords: Weight loss, Exercise, Weight reduction programs

*Correspondence: Benjamin.kelly@nuffieldhealth.com

'Nuffield Health Research Group, Nuffield Health, Ashley Avenue, Epsom,

Surrey KT18 5AL, UK

${ }^{2}$ Faculty of Health, Psychology and Social Care, Manchester Metropolitan

University, Manchester M15 6GX, UK

Full list of author information is available at the end of the article

(c) The Author(s). 2019 Open Access This article is distributed under the terms of the Creative Commons Attribution 4.0 International License (http://creativecommons.org/licenses/by/4.0/), which permits unrestricted use, distribution, and reproduction in any medium, provided you give appropriate credit to the original author(s) and the source, provide a link to the Creative Commons license, and indicate if changes were made. The Creative Commons Public Domain Dedication waiver (http://creativecommons.org/publicdomain/zero/1.0/) applies to the data made available in this article, unless otherwise stated. 


\section{Background}

Responsible for nearly two thirds of deaths worldwide [1], non-communicable diseases (NCD's) represent a significant global risk. In the UK, the probability of dying between the ages of 30 and 70 years from one of the four main NCDs is estimated to be $~ 12 \%$ [2]. In England, it is estimated that at least 1 in 20 people has type-2diabetes (T2DM) [3], with future trends predicting to rise by nearly a third to over four million diagnosed cases by 2025 [4]. In Scotland alone, where two thirds of adults are either overweight or obese, almost $20 \%$ of adults over the age of 16 years have some form of cardiovascular disease or T2DM [5].Obesity places a significant financial burden upon the National Health Services (NHS). Increased incidence of obesity parallels that of NCD's, with excess weight contributing to approximately $44 \%$ of the global T2DM burden [6]. With co-morbidities such as hypertension [7] and dyslipidaemia [8], augmented by obesity, there is a pressing need for effective interventional strategies. Previous projections have suggested that reducing body mass index (BMI) by $1 \%$ across the UK population (equivalent to 1 $\mathrm{kg}$ weight loss) would substantially reduce burden, saving up to 202,000 incident cases of T2DM and 122,000 cases of cardiovascular diseases over 20 years [9].

Guidelines recommend that primary care physicians in Britain identify people with obesity and offer clinical management [10] but few options for treatment exist in traditional primary care settings. Data from NHS-led interventions is sparse. Research has suggested that the 12-week 'Size down Programme'; an NHS group-based programme led by food advisers recruited from the local community, achieves significant weight loss, similar to competitor groups (Weight Watchers, Slimming World, Rosemary Conley, All $n=100$ ) [11]. The NHS also provides a free, online, self-help tool [12]. This weight loss plan was developed in association with The British Dietetic Association, and according to the NHS website, has been downloaded more than 4 million times as of August 2019. The plan involves downloading and following 12 weekly information packs which provide advice on both healthy eating and physical activity covering topics including: portion control, building-up physical activity, food swaps, comfort eating and long-term maintenance. Although this calorierestricted diet plan can be expected to be effective if adhered to, it remains uncertain whether an online programme will be sufficiently motivating to ensure adherence. It has been suggested that supervised diet plans have a greater chance of establishing and maintaining weight loss [13]. To our knowledge, the NHS weight loss plan has not previously been validated in any cohort, so questions remain about the effectiveness of this intervention.
To improve motivation and adherence, many commercial programmes share a similar structure of onceweekly group sessions ranging from 60 to $90 \mathrm{~min}$. Educational content within commercial programmes is predominantly focussed on dietary manipulation and tracking, with energy deficit the central physiological component to weight loss. Generally, activity is encouraged and is usually guided via measurable metrics such as steps. Jebb and colleagues [14] demonstrated that following a 12-month commercial weight loss intervention participants had increased odds of losing 5 and $10 \%$ of initial body weight in comparison to participants receiving standard care (weight loss advice from a primary care professional following national guidelines of the country of the participant; UK, Germany or Australia). A large $(N=29,326)$ participant data set from NHS referrals to a commercial weight loss programme identified that $57 \%$ of participants lost at least $5 \%$ of initial body weight with $12 \%$ losing $10 \%$ of initial body weight after just 12 sessions [15], with a number of studies replicating comparable findings across similar if not identical commercial interventions [16-20]. The 'Healthy Weight Program' (HWP) is a commercial, individually tailored 12-week intervention that provides both exercise and nutritional support. Dedicated face-to-face exercise coaching is delivered by personal trainers and face-toface nutrition coaching is delivered by registered nutritionists across multiple days of the week with the purpose of eliciting lifestyle and behaviour change to improve health and wellbeing in individuals. To date no research has compared a commercial intervention that combines intensive face-to-face support targeting both nutritional and exercise interventions to target weight loss with a less resource-intensive programmes such as the free online NHS weight loss tool.

The primary aim of the present study was to evaluate the effectiveness of reducing body mass through the scalable NHS resource compared to a commercial resource-intensive weight-loss intervention; as well as a 'no-advice' comparator group. The secondary aim of the present study was to evaluate the effectiveness of both interventions at improving health indicators related to obesity. We hypothesised that the HWP programme would result in significantly greater losses in body mass compared to the NHS weight loss programme. We further hypothesised that both programmes would elicit greater weight-loss than the 'no-advice' comparator group.

\section{Methods}

\section{Participants}

Participants for this parallel-randomized control trial were recruited from the local community near to the trial site (Glasgow, UK) via various advertising 
approaches including email, online features and social media in July and August 2016. People were directed to a purpose-built web page which provided greater detail, inclusion/exclusion criteria and an online contact form. Participant inclusion criteria included: aged between 18 and 50 years; a body mass index (BMI) between 30 and $45 \mathrm{~kg} / \mathrm{m}^{2}$; not currently regularly exercising assessed as 'low' via categorical scoring of the International Physical Activity Questionnaire [21]; not currently dieting nor have done so recently; not a current member of any Nuffield Health Fitness and Wellbeing centres; able to commit to visiting the trial sites 3-4 times per week for the duration of the intervention; not pregnant or lactating; not undergoing treatment for, possession or diagnoses of any metabolic or cardiovascular disease and previous surgical procedures for weight loss. Participants with controlled hypertension remained eligible for the trial. Individuals with a blood pressure (BP) of $>140 / 90$ $\mathrm{mmHg}$ were eligible to proceed upon approval and consent from their registered general practitioner. Prescreening of participants took place in July and August 2016 with the interventions taking place from September 2016 until January 2017 depending upon participant start date.

Following initial screening, 76 participants (26 male, 50 female) were invited to proceed to the intervention stage and provided written informed consent. Participants were block randomised by computer programme (https://www.randomizer.org) to one of three interventions: Healthy Weight Programme ${ }^{\text {mi }}$ (HWP), National Health Service programme (NHS), or gym only comparator group (GYM). Due to the nature of the intervention, blinding was not possible. The University of Bath Research Ethics Advisory Committee approved this study (ref: EP 15/16259/283). Following completion of the intervention phase, participants in all three groups were given full access to a Nuffield Health Fitness \& Wellbeing Centre for 12 months to assist them in achieving and maintaining any reduction in body mass and as a reimbursement for their time during the intervention. The CONSORT reporting guidelines were used [22].

\section{Healthy weight Programme}

The 12-week HWP intervention consisted of ten nutrition coaching sessions and 20 exercise sessions. The ten 1-h nutrition sessions were delivered by a registered nutritionist and consisted of a mix of one-to-one appointments and group classes. Two sessions were reserved for individual progress evaluations, specifically at weeks 6 and 10. Core themes included hunger and portions size, emotional eating, effects of sleep and stress, fuelling exercise, common weight loss myths, snacking, goal setting and meal planning. In addition, qualified exercise professionals provided twice-weekly exercise sessions for 10 weeks, starting at $30 \mathrm{~min}$ per session and increasing to $45 \mathrm{~min}$ by the end of the trial. Like the nutrition intervention, 2 weeks were reserved for individual progress evaluations. Sessions included indoor cycling, body weight circuit training, body pump classes and highintensity interval training. Outside of structured fitness sessions, participants had free access to the swimming pool, gym and fitness classes but were not allowed to access personal training other than what was provided as part of the intervention.

\section{NHS Programme}

The 12-week NHS intervention is an entirely selfmanaged online resource [12]. Participants were encouraged to utilise the broader NHS choices network and associated online tools and apps such as recipe finder, meal planner, calorie tracker and a moderated weight loss social forum. In brief, this intervention involved participants downloading weekly modules from the NHS website within which standardised tasks and guidance were detailed. Content included information on fibre consumption, portion control, exercise preparation, building-up physical activity, motivation strategy, breakfast advice, workplace wellbeing, cravings, alcohol awareness, plateaus, peer pressure, dining out, foreign foods, breaking down perceived barriers to change, food swaps, comfort eating and long-term maintenance. Participants received a 2-weekly call from the research team to resolve any technical/access issues. At no stage was additional coaching or feedback provided. For reasons of health and safety, participants were provided with an induction to the gym, and given full access to the gym and swimming pool for the duration of the intervention but were not allowed to access personal training during this time. Participants were familiarised with the basic American College of Sports Medicine (ACSM) physical activity guidelines [23] and how this would translate to the fitness and wellbeing centre by way of a handout (Appendix 2).

\section{Gym only}

The gym only group received no guidance or formal intervention. Following baseline assessments, participants were provided with an induction to the gym which acted as a health and safety measure. Participants were familiarised with the basic ACSM physical activity guidelines [23] and how this would translate to the fitness and wellbeing centre by way of a handout (Appendix 2) but were given no additional advice thereafter.

\section{Data collection}

All data were collected at baseline and 12 weeks and were taken following a $12 \mathrm{~h}$ overnight fast. Blood 
analyses were done using venous blood samples collected via venepuncture of the antecubital vein. Blood samples were collected into vacutainers ${ }^{\mathrm{TM}}$ (Becton Dickinson, Plymouth, UK; SST ${ }^{\mathrm{Ts}}$ II / 2KEDTA) for analysis of plasma insulin, blood lipid profile, fasting blood glucose and $\mathrm{HbA} 1 \mathrm{C}$. All samples were temporarily stored at $4{ }^{\circ} \mathrm{C}$ and analysed within $24 \mathrm{~h}$ of collection. Plasma total cholesterol and triglycerides (free glycerol blank subtracted) were measured enzymatically using established clinical chemistry laboratory methods [24, 25] (Nuffield Health, Glasgow, UK). High-density lipoprotein cholesterol (HDL-C) was measured by liquid selective detergent followed by enzymatic determination of cholesterol [26]. Low-density lipoprotein cholesterol (LDL-C) was calculated according to Friedewald et al. [27]. Total plasma insulin in serum was measured by radioimmunoassay [28] and blood glucose was measured using a modification of the glucose oxidase/peroxidase method [29, 30]. A HPLC-ESI/MS approach was utilized to measure blood HbA1c concentrations [31]. An estimation of insulin resistance and $\beta$-cell function was provided via the homeostasis model assessment as described elsewhere (HOMA-IR [32],).

Anthropometric measurements were made according to the recommendations of the International Standards for Anthropometric Assessment (ISAK) [33]. Fat mass and fat-free mass were assessed by bioelectrical impedance analysis in accordance with manufacturer recommendations (Bodystat 1500, Bodystat Ltd., UK). Blood pressure and resting heart rate were measured via automated blood pressure cuff (Omron M3 Comfort, Omron Corporation, Japan) in accordance with the European Society of Hypertension guidance [34]. Mean arterial pressure (MAP) was recorded and defined as [ $2 \mathrm{x}$ diastolic) + systolic $] \div 3$. Assessment of cardiovascular disease risk and T2DM risk assessed using the QRisk2 risk calculator [35] and QDiabetes [36] risk calculator respectively.

\section{Statistical analysis}

Data are presented as mean \pm SD. Statistical analyses were performed using IBM SPSS Statistics 23. As the aim of the study was to establish differences in the effects of two interventions vs. a control condition rather than establishing the effect of treatment assignments per se, we chose to employ Per Protocol analysis rather than Intention to Treat analysis. The primary outcome measure was the change in body mass from baseline to follow-up. To detect differences between the effects of the interventions on body mass with a medium effect size of $\mathrm{f}=0.25$ we required 18 participants in each group to achieve a power of $95 \%$ and $\alpha=0.05$. To allow for drop out of participants during the study period we aimed to recruit a sample size of 25 participants in each group. A two-way mixed ANOVA (intervention x time) was performed to determine the effects of the interventions on the outcome measures, with the intervention $\mathrm{x}$ time interaction effect as the main statistic of interest. In the case of significant main effects, post-hoc comparisons were performed using Fishers LSD (i.e. uncorrected paired t-tests) since there is no inflation of type 1 error rates following a significant main effect when only three comparisons are being made [37]. Alpha was set at 0.05 .

\section{Results}

Of the 76 participants who started $(n=25,25,26$ for HWP, NHS and GYM respectively), 13 withdrew citing a declination to continue in the study $(n=7,4$, and 2 for HWP, NHS and GYM respectively) (see Appendix 1 for participant flow diagram). Table 1 presents the characteristics of all starting participants and participants who completed the study and were included in the Per Protocol analysis. There were no significant differences between groups at baseline.

Main effects of time were observed (Table 2), with reductions in body mass $(p<0.001)$, BMI $(p<0.05)$, waist $(p<0.001)$ and hip circumference $(p<0.001)$, absolute body fat $(\mathrm{kg}, p<0.001)$, fat free mass $(p<0.01)$, plasma triglycerides $(p<0.01)$, LDL-C $(p<0.01)$, total cholesterol:HDL cholesterol ratio $(p<0.05)$, fasting blood glucose $(p<0.05)$, HbA1C $(p<0.05)$, and 10-year cardiovascular disease risk according to the QRISK-2 score $(p<0.01)$, and an increase in plasma HDL-C $(p<$ $0.01)$. No changes from pre- to post-intervention were observed for percentage body fat, total cholesterol, insulin, HOMA-IR, and T2DM risk (Q-Diabetes). A significant intervention $\mathrm{x}$ time interaction effect was observed for both body mass $(p<0.01)$ and BMI $(p<0.05)$. Greater reductions in body mass were observed in HWP $(5 \%, p<0.001)$ and NHS $(4 \%, p<0.001)$ compared to GYM (1\%) with no difference between the HWP and NHS interventions (Fig. 1).

\section{Discussion}

The primary aim of this study was to evaluate the effectiveness of a resource-intensive commercial weight-loss programme versus a free online NHS weight-loss intervention and an active control condition. We hypothesised that the more resource-intensive commercial HWP intervention would result in superior reductions in body mass compared to the NHS intervention and GYM control. However, despite the low cost and ease of delivery, the free, online NHS intervention was comparable to the commercially available, resource-intensive HWP intervention in reducing body mass, with significantly greater losses in body mass observed in both interventions compared to the active-control GYM condition. 
Table 1 Participant Characteristics

\begin{tabular}{|c|c|c|c|c|c|c|}
\hline & \multicolumn{2}{|l|}{ HWP } & \multicolumn{2}{|l|}{$\mathrm{NHS}$} & \multicolumn{2}{|l|}{ GYM } \\
\hline & $\begin{array}{l}\text { Starting Cohort } \\
N=25\end{array}$ & $\begin{array}{l}\text { Per Protocol } \\
\text { Analysis } N=18\end{array}$ & $\begin{array}{l}\text { Starting Cohort } \\
N=25\end{array}$ & $\begin{array}{l}\text { Per Protocol } \\
\text { Analysis } N=21\end{array}$ & $\begin{array}{l}\text { Starting Cohort } \\
N=26\end{array}$ & $\begin{array}{l}\text { Per Protocol } \\
\text { Analysis } N=24\end{array}$ \\
\hline Men / women & $10 / 15$ & $8 / 10$ & $8 / 17$ & $7 / 14$ & $8 / 18$ & $7 / 17$ \\
\hline Age (y) & $40 \pm 8(20-50)$ & $43 \pm 5(33-50)$ & $37 \pm 8(23-50)$ & $37 \pm 8(23-50)$ & $38 \pm 7(20-47)$ & $37 \pm 8(20-47)$ \\
\hline Height (m) & $\begin{array}{l}1.72 \pm 0.10 \\
(1.57-1.91)\end{array}$ & $\begin{array}{l}1.72 \pm 0.10 \\
(1.57-1.86)\end{array}$ & $\begin{array}{l}1.70 \pm 0.11 \\
(1.52-1.87)\end{array}$ & $\begin{array}{l}1.71 \pm 0.11 \\
(1.52-1.90)\end{array}$ & $\begin{array}{l}1.68 \pm 0.09 \\
(1.54-1.87)\end{array}$ & $\begin{array}{l}1.68 \pm 0.09 \\
(1.54-1.87)\end{array}$ \\
\hline Body Mass (kg) & $\begin{array}{l}106.06 \pm 15.66 \\
(82.50-141.00)\end{array}$ & $\begin{array}{l}106.38 \pm 14.90 \\
(89.50-140.00)\end{array}$ & $\begin{array}{l}102.53 \pm 16.79 \\
(77.00-145.10)\end{array}$ & $\begin{array}{l}103.13 \pm 16.92 \\
(77.00-145.10)\end{array}$ & $\begin{array}{l}98.52 \pm 13.32 \\
(80.20-126.80)\end{array}$ & $\begin{array}{l}98.98 \pm 13.54 \\
(80.20-126.80)\end{array}$ \\
\hline $\mathrm{BMI}\left(\mathrm{kg} / \mathrm{m}^{2}\right)$ & $\begin{array}{l}36.01 \pm 3.26 \\
(30.00-41.20)\end{array}$ & $\begin{array}{l}35.86 \pm 3.42 \\
(29.67-40.47)\end{array}$ & $\begin{array}{l}35.31 \pm 3.40 \\
(29.71-41.76)\end{array}$ & $\begin{array}{l}35.25 \pm 3.51 \\
(29.71-40.19)\end{array}$ & $\begin{array}{l}34.78 \pm 2.90 \\
(30.07-40.11)\end{array}$ & $\begin{array}{l}34.85 \pm 2.97 \\
(30.07-40.11)\end{array}$ \\
\hline
\end{tabular}

Values shown are means \pm SD (range)

This is the first study to demonstrate the effectiveness of the free online NHS weight loss programme at enabling individuals to achieve reductions in body mass. The NHS resource has several useful additions on the associated web-page, including: automated support email signup, access to a weight-loss forum, a calorie-checker, a mobile app, and articles on success stories and tips to overcoming barriers. This volume of additional support options and the ability to access supportive content at the participants' leisure may explain the similarities in results of the NHS intervention compared to the HWP intervention, which does not provide access to such content outside of the face-to-face sessions, although usage of additional content was not assessed in the current study. A large volume of content including exercise videos is available at the Nuffield Health website, but participants were not specifically made aware of this.

The UK Department of Health's best practice guidance for weight management [38] advises that weight loss programmes must achieve measurable health outcomes. Specifically, weight loss programmes should ensure that interventions lead to an average weight loss of at least $3 \%$, with at least $30 \%$ of participants losing at least $5 \%$ of their initial weight. This target was met in the HWP and NHS group, which further demonstrates the effectiveness of the NHS weight loss plan. Whilst both HWP and NHS groups achieved 5\% weight loss in 50 and 38\% of participants respectively, the GYM group marginally missed this target, achieving 3\% weight loss in $29 \%$ of participants, which may suggest that the observed effects in the NHS and HWP groups was not merely because of offering free gym access.

Heshka and colleagues [17] previously demonstrated that weight loss was significantly greater following a commercial weight loss programme (Weight Watchers; $-4 \mathrm{~kg})$ compared to a self-help programme $(-1.5 \mathrm{~kg})$. This is at odds with the current findings, with the commercial HWP group achieving $5.2 \pm 4.2 \mathrm{~kg}$ weight loss (5\%) and the NHS self-help group achieving $4.2 \pm 5.5 \mathrm{~kg}$ weight loss (4\%) with no statistical difference between the two groups. A study by Baetge et al. [39] compared a programme that incorporates both exercise and dietary interventions (Curves) versus programmes that provide a dietary intervention only but advocates for exercise (Weight Watchers, Jenny Craig and Nutrisystem) and a non-intervention control group, evaluated over a 12week period in a population of participants who were overweight or obese. Data demonstrated significant reductions in body mass versus a no-intervention control for all groups. Briefly, these were translated in to the following percentage changes: Curves (a combined mealplan and exercise intervention) $-4.7 \%$, Weight Watchers (a group based, points-orientated nutritional intervention) $-4.8 \%$, Jenny Craig at Home (an online nutritionfocussed intervention) $-5.9 \%$, and Nutrisystem Advance Select (a meal-plan based system with online support) $-5.3 \%$. The results of this study align to those of the present study where a combined exercise and nutrition intervention (HWP) was not superior to a nutrition intervention which advocates for exercise (NHS). Furthermore, a systematic review and meta-analysis of 26 studies examining remotely delivered standalone interventions to elicit eating behaviour change by Teasdale et al [40] found a small but significant positive effect on eating behaviour change compared to control groups. The authors conclude that, albeit small, standalone selfmanagement or targeted feedback interventions - such as the online NHS programme - could have an appreciable impact at a population level.

A time $\mathrm{x}$ intervention interaction only existed for body mass and BMI; however, a main effect of time showed positive changes in several additional indices including: waist circumference, HDL cholesterol, LDL cholesterol, plasma triglycerides, and blood glucose. These findings suggest that all three conditions elicited an improvement in several health markers however the two main interventions were not superior to the active-control condition for any health marker out with body mass and BMI. 


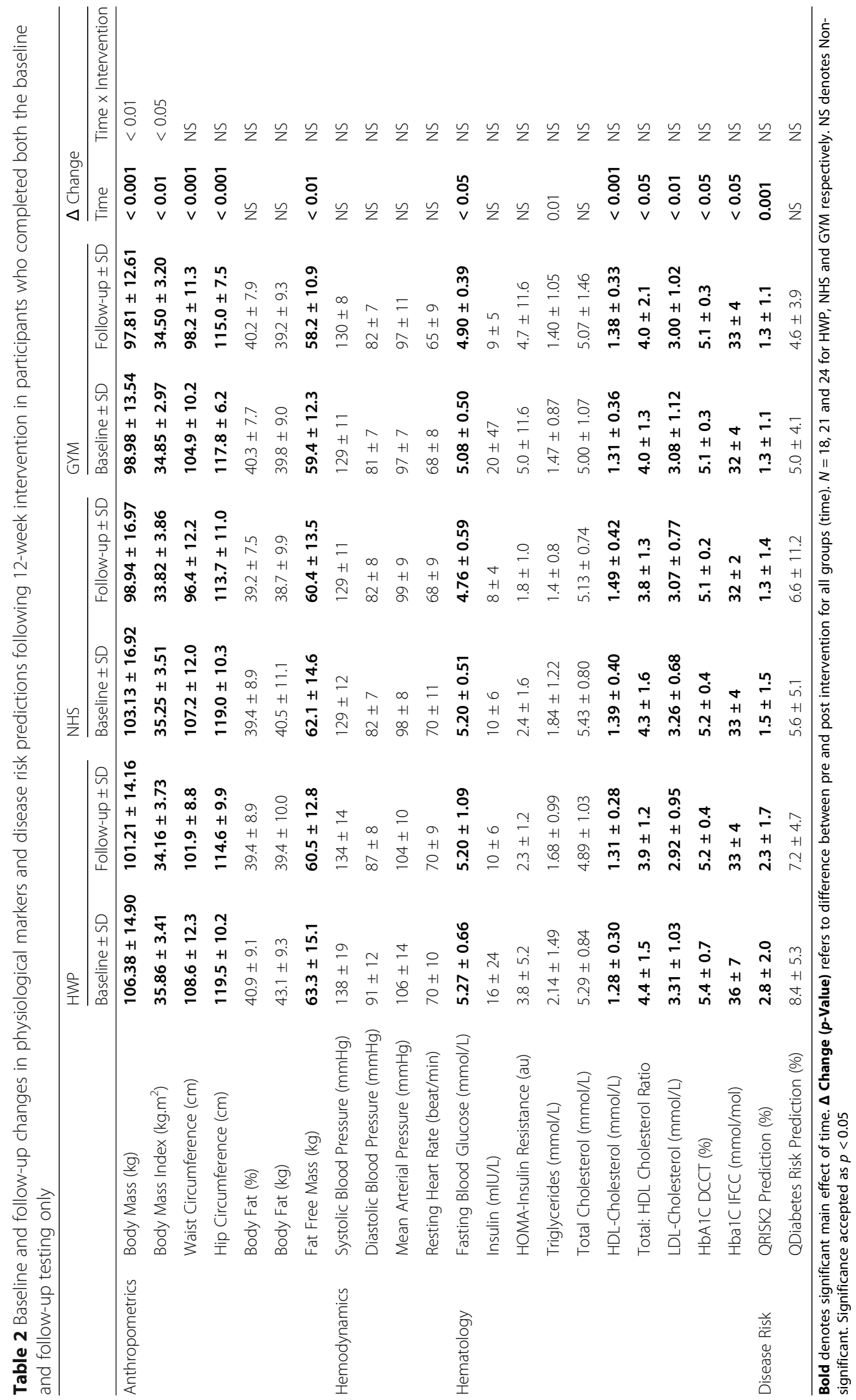




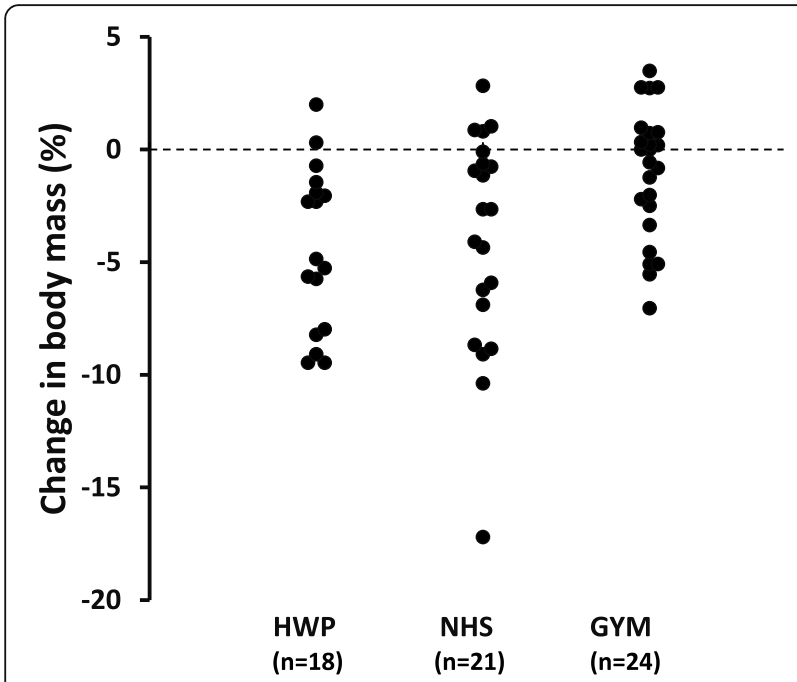

Fig. 1 Individuals' post intervention changes in body mass between groups

Results in the present study are at odds with Baetge and colleagues [39] who showed significant pre to post improvements in the aforementioned following 12-weeks of weight-loss interventions compared to their control group. The present study and that of Baetge et al. [39] differ somewhat however in that the present study includes both males and females and an active control condition allowing for the assessment of both weightloss interventions in a 'real-world' setting whereas Baetge and colleagues recruited women only and had a no-intervention control. In the case of the present study, although the primary aim was achieved, it is possible to postulate that the inclusion of the "real-world" control condition masked further between-group differences seen in other studies that used no-intervention control groups.

Current guidance from the National Institute Health and Care Excellence [41] recommends that individuals who are overweight or obese be referred to group rather than individual programmes due to cost effectiveness. This can include lifestyle weight management programmes delivered by the public, private or voluntary sector. The NHS intervention in the current study offers a potential opportunity that is scalable, cost effective and can acutely achieve clinically significant weight loss similar to commercial lifestyle weight management programmes that patients are referred to such as Weight Watchers [15] and slimming world.

\section{Strengths and limitations}

The addition of an active-control group was a strength of the present study. We recruited a very motivated cohort who were randomised to three groups with varying levels of support. Providing the control group with free access to fitness facilities but no further support allowed for the evaluation of both interventions in a 'real-world' setting.

The duration of the present study (12 weeks) is acute and therefore longer-term outcomes are not known. A 6 and 12 -month follow-up was planned but was unable to be conducted due to operational constraints within the delivery organisation. This was unfortunate as a key question about the long-term maintenance of weightloss following both interventions remains unanswered. Future work requires detailed objective monitoring of physical activity. Participants in the present study were given access to fitness facilities however an unforeseen error meant that usage data was not collected. The NHS resource has several additions on the associated webpage; although participants were made aware of this information, we were unable to track usage. It should also be noted that the results in the present study may be explained by the fact that the present study had a very motivated cohort given that participants volunteered to take part and therefore, results in the general population may differ.

The present study was too small to derive reliable estimates of adherence, and as such we chose to initially examine the effectiveness of the two interventions at inducing reductions in body mass. Now that we have established that the free, online NHS programme does not result in significantly lower reductions in body mass compared to a resource-intensive commercial programme, there is a need for future studies to establish possible differences in adherence and to utilise Intention to Treat analysis rather than Per Protocol analysis as used in the present study [42].

\section{Conclusion}

This is the first study to evaluate the free online NHS self-help weight-loss tool and compare it to a commercial weight loss programme. Our findings suggest that the NHS weight loss tool is an effective intervention for reducing body mass in the short term, and that providing a more resource-intensive intervention does not necessarily yield additional benefits, at least in the short term. Both interventions were superior compared to the control group at eliciting a reduction in body mass. Although current guidelines regarding weight-loss recommend people are referred to group programmes due to their cost-effectiveness, the current study demonstrates clinically significant weight-loss can be achieved by a free, online, scalable, self-help website. Thus, clinicians and the NHS may have an alternative and effective option to support weight-loss, with potential additive benefits expected should a structured exercise environment be made available. 


\section{Appendix 1}

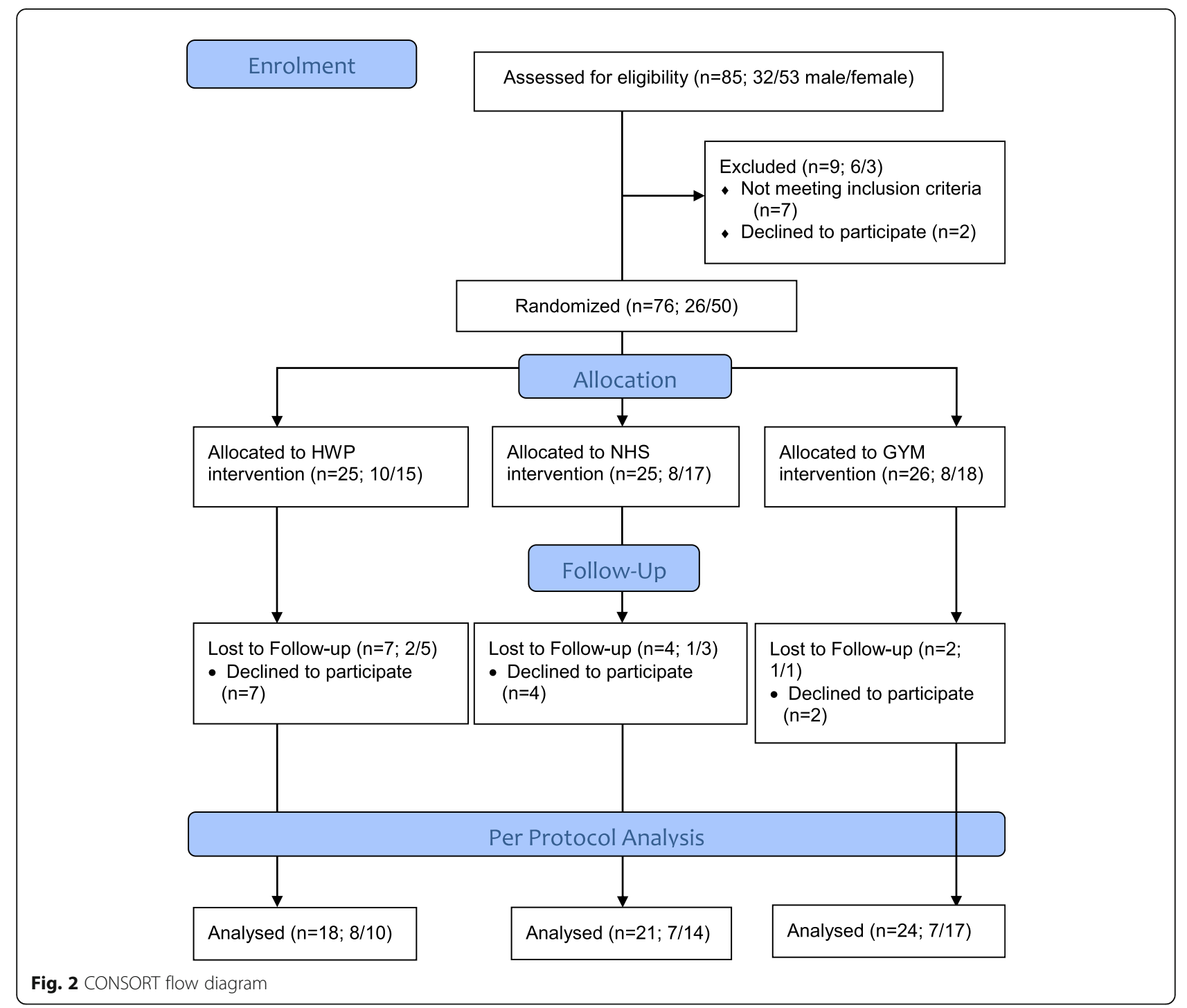

\section{Appendix 2}

\section{Gym-based Exercise Information Guide}

Healthy Weight Study $\backslash$ Nuffield Health Research Group

The American College of sports Medicine (ACSM) recommend that each person performs: $150 \mathrm{~min}$ of moderate of $75 \mathrm{~min}$ of vigorous intensity aerobic exercise across 7 days, as well as 2 sessions of whole body resistance exercises and at least 2 sessions working on flexibility.

\section{0 min of moderate intensity or 75 min of vigorous intensity aerobic exercise per week}

- Aerobic exercise improves your hearts efficiency as a pump and when performed consistently, can reduce your risk of virtually all lifestyle related diseases.
Moderate intensity - a 4-7 out of 10 in terms of perceived effort. You are likely to have increased breathing rate, perhaps be lightly sweating; however, you should be able to keep this intensity up for around an hour.

$\bigcirc$ Vigorous intensity - an 8 out of 10 at least in terms of intensity. You shouldn't feel comfortable at this intensity. You're likely to be sweating heavily and short of breath; however, you should be able to speak in short phrases.

Example Moderate Intensity Exercises

- Continuous swimming

- Brisk Walking/light jogging

- Continuous cycling/stepper/rowing/cross trainer

Example Vigorous Intensity Exercises 
- Body Pump class

- Metafit Class

- Cycle Class

- Interval training on cardio machines

- Circuits Class

- Boxing Fit Class

\section{Whole body resistance training at least twice per week:}

- Resistance training typically involves performing exercises against a force or weight for several repetitions per set. There are many variations of repetitions and sets but the general principles you should follow are:

$\bigcirc$ Perform at least 1 exercise per large muscle group such as:

- Leg Press Targets most muscles in your legs

- Chest Press Targets most muscles in your chest as well as arms

- Seated row Targets muscles in your upper and middle back and arms

$\bigcirc$ You can utilise fixed weight machines or freeweights depending on your experience. If you are inexperienced, fixed-weight machines will guide you safely.

$\bigcirc$ Keep track of the exercises you do, the weights, the repetitions and the sets you perform with a view to increased one of these aspects each week to continuously improve.

$\bigcirc$ Performing 3 sets of $12-15$ repetitions will be sufficient to start with but you can increase/ decrease the sets/reps as you feel the need to, but keep a note so you can improve next time you're in the gym

Some fitness classes will also count as resistance training such as body pump and circuits.

\section{Perform at least two sessions of flexibility exercises per week:}

- Working to improve and then maintain flexibility across all major muscle groups at least twice per week will help improve and maintain range of motion in your joints and may help reduce certain risks such as low back pain.

$\bigcirc$ Performing a yoga class is a great way to learn various stretches which you can then implement into your workout routine

$\bigcirc$ You can attend yoga classes for free during the study.

Additional Information: For the duration of the study, you will be unable to purchase Personal Training. For any questions or issues, please contact one of the researchers.

\section{Abbreviations}

ACSM: American College of Sports Medicine; BMI: Body mass index; BP: Blood pressure; GYM: Gym-only comparator group; HDL-C: High-density lipoprotein cholesterol; HOMA-IR: Homeostatic modelling assessment for insulin resistance; HWP: Healthy Weight Programme; LDL-C: Low-density lipoprotein cholesterol; MAP: Mean arterial pressure; NCD's: Noncommunicable Diseases; NHS: National Health Service; T2DM: Type-2diabetes

\section{Acknowledgements}

Not Applicable.

\section{Authors' contributions}

$\mathrm{Al}, \mathrm{GT}, \mathrm{JK}$ and BK conceived and designed the original protocol. Al, GT, MC, $\mathrm{NV}, \mathrm{JK}$ and BK were involved in amending the protocol. Al coordinated the study throughout. Data collection and entry was carried out by Al, GT and BK. MC delivered the HWP intervention. NV analysed the data. Al wrote the initial draft of the manuscript with BK, NV and JK. All authors contributed to subsequent drafts and have read and approved the manuscript.

\section{Funding}

The study was funded through the Nuffield Health Charity (Registered Charity Numbers: 205533 in England and Wales and SC041793 in Scotland). This research was supported by the National Institute for Health Research (NIHR) Leicester Biomedical Research Centre. The views expressed are those of the authors and not necessarily those of the NHS, the NIHR, or the Department of Health. Authors AI, GT, MC and BK are employees of study funder Nuffield Health as declared in 'Competing Interests'. Their involvement is fully outlined in 'Authors' Contributions' below.

\section{Availability of data and materials}

The data generated and/or analysed during the current study are available from the corresponding author on reasonable request.

\section{Ethics approval and consent to participate}

The University of Bath Research Ethics Advisory Committee approved this study on 22nd July 2016 (ref: EP 15/15 259/283). Written informed consent was gained from all participants prior to their enrolment into this study.

\section{Consent for publication}

Not applicable.

\section{Competing interests}

Authors NBJ Vollaard and JA King report no conflict of interest however both are voluntary members of Nuffield Health's External Research Advisory Board. Authors AQ Innes, G Thomson, M Cotter and BM Kelly report personal fees from Nuffield Health outside of the submitted work.

\section{Author details}

${ }^{1}$ Nuffield Health Research Group, Nuffield Health, Ashley Avenue, Epsom, Surrey KT18 5AL, UK. 'Faculty of Health, Psychology and Social Care, Manchester Metropolitan University, Manchester M15 6GX, UK. ${ }^{3}$ National Centre for Sport and Exercise Medicine, Loughborough University, Loughborough LE11 3TU, UK. ${ }^{4}$ National Institute for Health Research Leicester Biomedical Research Centre, Leicester LE3 8QD, UK. ${ }^{5}$ Faculty of Health Sciences and Sport, University of Stirling, Stirling FK9 4LA, UK.

Received: 12 August 2019 Accepted: 11 December 2019

Published online: 23 December 2019

\section{References}

1. Bloom DE, Cafiero E, Jané-Llopis E, Abrahams-Gessel S, Bloom LR, Fathima S, et al. The global economic burden of noncommunicable diseases. Program on the Global Demography of Aging; 2012

2. World Health Organization. World Health Organization - Noncommunicable Diseases (NCD) Country Profiles for the United Kingdom. 2014. Available from: http://www.who.int/nmh/countries/gbr_en.pdf?ua=1

3. Diabetes UK. Diabetes: facts and stats. Diabetes UK. 2014

4. DIABETES UK. State of the Nation 2012 England. Report Key statistics on diabetes. 2012. Available from: https://www.diabetes.org.uk/resources-s3/201 7-11/state-of-the-nation-2012.pdf 
5. McLean J, Christie S, Gray L. Scottish health survey 2016: volume 1: Main report; 2017.

6. World Health Organization. Global Health Risks-Mortality and burden of disease attributable to selected major risks. 2017. Available from: https://www.who.int/ healthinfo/global_burden_disease/GlobalHealthRisks_report_full.pdf

7. Colosia AD, Palencia R, Khan S. Prevalence of hypertension and obesity in patients with type 2 diabetes mellitus in observational studies: a systematic literature review. Diabetes Metab Syndr Obes targets Ther. 2013;6:327.

8. Franssen R, Monajemi H, Stroes ESG, Kastelein JJP. Obesity and dyslipidemia. Med Clin North Am. 2011:95(5):893-902.

9. Wang YC, McPherson K, Marsh T, Gortmaker SL, Brown M. Health and economic burden of the projected obesity trends in the USA and the UK. Lancet. 2011;378(9793):815-25.

10. National Institute for Health and Care Excellence. Obesity: the prevention, identification, assessment and management of overweight and obesity in adults and children. 2006; Available from: https://www.nice.org.uk/guidance/ cg189/evidence/obesity-update-appendix-m-pdf-6960327447

11. Jolly K, Lewis A, Beach J, Denley J, Adab P, Deeks JJ, et al. Comparison of range of commercial or primary care led weight reduction programmes with minimal intervention control for weight loss in obesity: lighten Up randomised controlled trial. BMJ. 2011;343(nov03_2):d6500 Available from: http://www.bmj.com/content/343/bmj.d6500.

12. NHS Choices Weight Loss Guide [Internet]. Available from: https://www.nhs. uk/Livewell/weight-loss-guide/Pages/losing-weight-getting-started.aspx

13. Lemstra M, Bird $Y$, Nwankwo C, Rogers M, Moraros J. Weight loss intervention adherence and factors promoting adherence: a meta-analysis. Patient Prefer Adherence. 2016;10:1547.

14. Jebb SA, Ahern AL, Olson AD, Aston LM, Holzapfel C, Stoll J, et al. Primary care referral to a commercial provider for weight loss treatment versus standard care: a randomised controlled trial. Lancet. 2011:378(9801):1485-92.

15. Ahern AL, Olson AD, Aston LM, Jebb SA. Weight watchers on prescription: an observational study of weight change among adults referred to weight watchers by the NHS. BMC Public Health. 2011;11(1):434.

16. Lavin JH, Avery A, Whitehead SM, Rees E, Parsons J, Bagnall T, et al. Feasibility and benefits of implementing a slimming on referral service in primary care using a commercial weight management partner. Public Health. 2006;120(9):872-81.

17. Heshka S, Anderson JW, Atkinson RL, Greenway FL, Hill JO, Phinney SD, et al. Weight loss with self-help compared with a structured commercial program: a randomized trial. Jama. 2003;289(14):1792-8.

18. Truby H, Baic S, Fox KR, Livingstone MBE, Logan CM, Macdonald IA, et al. Randomised controlled trial of four commercial weight loss programmes in the UK: initial findings from the BBC "diet trials". Bmj. 2006;332(7553):1309-14

19. Rock CL, Pakiz B, Flatt SW, Quintana EL. Randomized trial of a multifaceted commercial weight loss program. Obesity. 2007;15(4):939-49.

20. Tsai AG, Wadden TA. Systematic review: an evaluation of major commercial weight loss programs in the United States. Ann Intern Med. 2005;142(1):56-66.

21. Craig CL, Marshall AL, Sjorstrom M, Bauman AE, Booth ML, Ainsworth BE, et al. International physical activity questionnaire: 12-country reliability and validity. Med Sci Sports Exerc. 2003;35(8):1381-95.

22. Shulz RA, Yutzey KE. Calcineurin signalling and NFAT activation in cardiovascular and skeletal muscle development. Dev Biol. 2004;266(1):1-16.

23. Riebe D, Franklin BA, Thompson PD, Garber CE, Whitfield GP, Magal M, et al. Updating ACSM's recommendations for exercise preparticipation health screening. Med Sci Sports Exerc. 2015;47(11):2473-9.

24. Allain CC, Poon LS, Chan CSG, Richmond W, Fu PC. Enzymatic determination of total serum cholesterol Clin Chem 20: 470-475. Find this Artic online. 1974;

25. Simpson EJ, DeMers LM, Kreig AF. Faster enzymatic procedure for serum triglyceride. Clin Chem. 1975;21:1983-5.

26. Warnick GR, Albers JJ. A comprehensive evaluation of the heparinmanganese precipitation procedure for estimating high density lipoprotein cholesterol. J Lipid Res. 1978;19(1):65-76.

27. Friedewald WT, Levy RI, Fredrickson DS. Estimation of the concentration of low-density lipoprotein cholesterol in plasma, without use of the preparative ultracentrifuge. Clin Chem. 1972;18(6):499-502.

28. Morgan CR, Lazarow A. Immunoassay of insulin: two antibody system: plasma insulin levels of normal, subdiabetic and diabetic rats. Diabetes. 1963;12(2):115-26.

29. Lott JA, Turner K. Evaluation of Trinder's glucose oxidase method for measuring glucose in serum and urine. Clin Chem. 1975;21(12):1754-60.
30. Trinder $P$. Determination of blood glucose using an oxidase-peroxidase system with a non-carcinogenic chromogen. J Clin Pathol. 1969;22(2):158-61.

31. Jeppsson J-O, Kobold U, Barr J, Finke A, Hoelzel W, Hoshino T, et al. Approved IFCC reference method for the measurement of $\mathrm{HbA} 1 \mathrm{C}$ in human blood. Clin Chem Lab Med. 2002;40(1):78-89.

32. Matthews DR, Hosker JP, Rudenski AS, Naylor BA, Treacher DF, Turner RC. Homeostasis model assessment: insulin resistance and $\beta$-cell function from fasting plasma glucose and insulin concentrations in man. Diabetologia. 1985;28(7):412-9.

33. International Standards for Anthropometric Assessment. The International Society for the Advancement of Kinanthropometry; 2001.

34. Redon J, Narkiewicz K, Nilsson PM, Burnier M, Viigimaa M, Ambrosioni E, et al. 2013 ESH/ESC guidelines for the management of arterial hypertension. Eur Heart J. 2013;34:2159-219.

35. Hippisley-Cox J, Coupland C, Vinogradova Y, Robson J, May M, Brindle P. Derivation and validation of QRISK, a new cardiovascular disease risk score for the United Kingdom: prospective open cohort study. BMJ. 2007; 335(7611):136

36. Hippisley-Cox J, Coupland C, Robson J, Sheikh A, Brindle P. Predicting risk of type 2 diabetes in England and Wales: prospective derivation and validation of QDScore. Bmj. 2009;338:b880.

37. Howell DC. Statistical methods for psychology. Brooks/Cole; 1997.

38. Department of Health. Developing a specification for lifestyle weight management services Best practice guidance for tier 2 services. 2013. Available from: https://www.gov.uk/government/uploads/system/uploads/ attachment_data/file/142723/Weight_Management_Service_Spec_FINAL_ with_IRB.pdf

39. Baetge C, Earnest CP, Lockard B, Coletta AM, Galvan E, Rasmussen C, et al. Efficacy of a randomized trial examining commercial weight loss programs and exercise on metabolic syndrome in overweight and obese women. Appl Physiol Nutr Metab. 2017;42(2):216-27.

40. Teasdale N, Elhussein A, Butcher F, Piernas C, Cowburn G, Hartmann-Boyce $J$, et al. Systematic review and meta-analysis of remotely delivered interventions using self-monitoring or tailored feedback to change dietary behavior. Am J Clin Nutr. 2018;107(2):247-56. Available from. https://doi.org/ 10.1093/ajcn/nqx048.

41. National Institute for Health and Care Excellence. Weight Management: Lifestyle Services for Overweight or Obese Adults (PH53). London: National Institute for Health and Care Excellence; 2014. Available from: https://www. nice.org.uk/guidance/ph53/resources/weight-management-lifestyle-servicesfor-overweight-or-obese-adults-pdf-1996416726469

42. Hernán MA, Robins JM. Per-protocol analyses of pragmatic trials. N Engl J Med. 2017;377(14):1391-8.

\section{Publisher's Note}

Springer Nature remains neutral with regard to jurisdictional claims in published maps and institutional affiliations.

Ready to submit your research? Choose BMC and benefit from:

- fast, convenient online submission

- thorough peer review by experienced researchers in your field

- rapid publication on acceptance

- support for research data, including large and complex data types

- gold Open Access which fosters wider collaboration and increased citations

- maximum visibility for your research: over $100 \mathrm{M}$ website views per year

At $\mathrm{BMC}$, research is always in progress.

Learn more biomedcentral.com/submissions 